\title{
(2) OPEN ACCESS \\ Wegovy (semaglutide): a new weight loss drug for chronic weight management
}

\author{
Gurdeep Singh 이, ${ }^{1}$ Matthew Krauthamer, ${ }^{2}$ Meghan Bjalme-Evans ${ }^{3}$
}

${ }^{1}$ Internal Medicine, Our Hospital, Binghamton, New York, USA

${ }^{2}$ Emergency Medicine, Our Hospital, Binghamton, New York, USA

${ }^{3}$ Endocrinology, Our Lady of Lourdes Memorial Hospital, Binghamton, New York, USA

\section{Correspondence to}

Dr Gurdeep Singh, Our

Lady of Lourdes Memorial

Hospital, Binghamton, NY

13905, USA;

drgsingh803@gmail.com

Accepted 6 October 2021 Published Online First 27 October 2021 Lady of Lourdes Memorial Lady of Lourdes Memorial

\begin{abstract}
Obesity is a growing epidemic within the USA. Because weight gain is associated with an increased risk of developing life-threatening comorbidities, such as hypertension or type 2 diabetes, there is great interest in developing non-invasive pharmacotherapeutics to help combat obesity. Glucagon-like peptide-1 (GLP-1) receptor agonists are a class of antidiabetic medications that have shown promise in encouraging glycemic control and promoting weight loss in patients with or without type 2 diabetes. This literature review summarizes and discusses the weight loss results from the SUSTAIN (Semaglutide Unabated Sustainability in Treatment of Type 2 Diabetes), PIONEER (Peptide Innovation for Early Diabetes Treatment), and STEP (Semaglutide Treatment Effect in People with Obesity) clinical trial programs. The SUSTAIN and PIONEER clinical trials studied the use of $1.0 \mathrm{mg}$, once-weekly, subcutaneous and oral semaglutide (a new GLP-1 homolog), respectively, on participants with type 2 diabetes. The STEP trial examined the effects of $2.4 \mathrm{mg}$, once-weekly, subcutaneous semaglutide on patients with obesity. Trial data and other pertinent articles were obtained via database search through the US National Library of Medicine Clinical Trials and the National Center for Biotechnology Information. All three clinical trials demonstrated that semaglutide (injected or oral) has superior efficacy compared with placebo and other antidiabetic medications in weight reduction, which led to Food and Drug Administration approval of Wegovy (semaglutide) for weight loss.
\end{abstract}

\section{Linked InTRODUCTION}

- http://dx.doi.org/10.

1136/jim-2021-002227

- http://dx.doi.org/10.

1136/jim-2021-002268

Check for updates

(C) American Federation for Medical Research 2022.

Re-use permitted under CC BY-NC. No commercial re-use. Published by BMJ.

To cite: Singh $\mathrm{G}$, Krauthamer M, BjalmeEvans M. J Investig Med 2022;70:5-13.
The worldwide prevalence of overweight and obesity, defined as having a body mass index (BMI) between $25.0-29.9 \mathrm{~kg} / \mathrm{m}^{2}$ and $\geq 30.0 \mathrm{~kg} /$ $\mathrm{m}^{2},{ }^{1}$ has almost tripled since the 1970 s, with nearly $40 \%$ of adults being overweight and $13 \%$ obese, and these numbers have been steadily increasing. ${ }^{2}$ Patients with obesity are at an increased risk of developing comorbidities such as hypertension, type 2 diabetes, hyperlipidemia, stroke, and even certain cancers. ${ }^{34}$ Consequently, they have an increased risk of death due to cardiovascular complications, which is the most prevalent cause of death in patients with obesity. ${ }^{5}$ Another negative consequence is the cost of combating the obesity epidemic, estimated to take up around $16 \%-18 \%$ of the total health expenditures in the USA by $2030 .^{3}$

Guidelines for treating overweight and obesity in the USA place lifestyle modifications, including moderate to vigorous exercise, decreased caloric intake, and behavioral therapy, as the first steps in the intervention. ${ }^{1}$ Lifestyle modifications can reduce the risk of developing cardiovascular complications, but patients do not easily maintain any accomplished weight loss. ${ }^{1}$ If no significant changes occur through lifestyle modifications, adding pharmacotherapeutics may help to promote weight loss. ${ }^{1}$

There are four Food and Drug Administration (FDA)-approved medications on the market for weight loss, namely phentermine-topiramate (Qsymia), orlistat (Xenical), naltrexonebupropion (Contrave), and the glucagon-like peptide-1 receptor agonist (GLP-1 RA) liraglutide (Saxenda). ${ }^{13}$ Lorcaserin (Belviq) was previously given FDA approval but was recalled at the beginning of 2020 due to an increased risk of malignancies. ${ }^{6}$ Wegovy (semaglutide), the GLP-1 RA discussed in this article, received FDA approval in 2021.7

GLP-1 RAs have repeatedly shown promising results in the reduction of body weight in obese patients with and without diabetes. 3489 They are also successful at improving glycemic control by stimulating insulin secretion and inhibiting glucagon secretion without the onset of hypoglycemia. ${ }^{10}$ Although their weight loss effects are well known, the mechanism underlying these effects is still debatable. Most investigations into the underlying mechanisms of GLP-1 on appetite and weight loss have focused on liraglutide. ${ }^{1}$ The most notable known mechanisms are linked with the central and peripheral nervous systems through direct activation of the hypothalamus and hindbrain or indirect activation via the vagus nerve, resulting in reduced appetite and food intake. ${ }^{3}$ Projections from the nucleus tractus solitarius in the hindbrain to the ventral tegmental area and the nucleus accumbens can implicate GLP-1 in influencing reward and motivation reactions toward food as well, reducing general palatability. ${ }^{3}$ GLP-1 RAs are also known to delay gastric emptying, but the effect on patients' total weight loss seems to be minimal. ${ }^{3}$ Overall, the mechanisms appear to affect energy intake and not resting metabolic rate. ${ }^{8}$ 
Studies have also investigated the gastrointestinal (GI) side effects, namely nausea and vomiting, reported by trial participants and whether or not these have contributed to the weight loss effects of GLP-1 RAs in any manner. Based on the SUSTAIN clinical trial program data, nausea and vomiting were minimally related to patients' weight loss. ${ }^{8}$ The trial cited here also evaluated the primary causes of weight loss in patients taking semaglutide (Ozempic), a relatively new GLP-1 RA currently on the market only as an antidiabetic drug in both injectable and oral tablet forms. A lower energy intake was considered the key factor responsible for bodyweight reduction. ${ }^{8}$ The Semaglutide Treatment Effect in People with Obesity (STEP) program is a phase III clinical trial program focused on the approval of semaglutide as a weight loss medication in patients with obesity. This program included five trials primarily focused on comparing $2.4 \mathrm{mg}$, once-weekly, subcutaneous semaglutide with placebo treatment. ${ }^{11-15}$ This trial program did not compare semaglutide with other antiobesity medications currently on the market. Results have shown that $2.4 \mathrm{mg}$ semaglutide, when used in combination with lifestyle modifications, shows clinically significant weight loss in patients with obesity compared with placebo. ${ }^{11-15}$

Semaglutide not only showed improvement in diabetes and body weight but also lowered the rate of cardiovascular death, non-fatal myocardial infarction, or non-fatal stroke in patients with diabetes mellitus type 2 at high risk of cardiovascular disease. ${ }^{16}$

\section{METHODS}

All pertinent journal entries used to create this review were obtained via search with the National Center for Biotechnology Information database. Two search items, semaglutide and obesity, were used. There were 127 results received but only SUSTAIN (Semaglutide Unabated Sustainability in Treatment of Type 2 Diabetes), PIONEER (Peptide Innovation for Early Diabetes Treatment), and STEP (Semaglutide Treatment Effect in People with Obesity) trials were reviewed because these were original, randomized, controlled clinical trials done before approval of semaglutide for management of diabetes and obesity.

Three of the 13 total trials comprising the SUSTAIN program were not included in this review due to redundancy of the medications' comparisons. ${ }^{17-19}$ The most recent literature search was performed in July 2021.

\section{SUMMARY OF SUSTAIN EVIDENCE}

The recent SUSTAIN (Semaglutide Unabated Sustainability in Treatment of Type 2 Diabetes), clinical trial program investigated the safety and efficacy of subcutaneous semaglutide, which has a 94\% homology with endogenous GLP-1. ${ }^{10}$ The addition of a fatty diacid chain at position 26 improves albumin binding. The substitution of alanine for $\alpha$-aminoisobutyric acid at position 8 makes the molecule less susceptible to degradation by dipeptidyl peptidase- 4 . These modifications have resulted in an increased half-life of the molecule, allowing subcutaneous semaglutide to be administered once weekly. ${ }^{10}$

Throughout the 10 studies reviewed here (table 1), semaglutide was found to be superior at reducing hemoglobin A1c (HA1c) and reducing participants' body weight when compared with placebo ${ }^{16} 20-22$ and with multiple antidiabetic medications, including insulin glargine, ${ }^{23}$ sitagliptin,${ }^{8}$ exenatide extended release (ER), ${ }^{10}$ dulaglutide, ${ }^{24}$ canagliflozin, ${ }^{25}$ and liraglutide. ${ }^{26}$ Also, the superiority of GLP-1 RAs at promoting weight loss and glycemic control has been shown in a previous clinical trial investigating the safety and efficacy of other medications within this drug class. ${ }^{4}$ However, with this similarity in mind, it is essential to note that the magnitude of weight loss experienced by participants on semaglutide versus comparators was greater than the weight loss found in other GLP-1 RA clinical trial programs. ${ }^{4}$ Compared with these other GLP-1 RAs, semaglutide was superior at promoting bodyweight reduction in the SUSTAIN clinical trial (table 1). Comparing other long-acting GLP-1 RAs with semaglutide, namely exenatide $\mathrm{ER}^{10}$ and dulaglutide, ${ }^{24}$ semaglutide showed superiority in overall weight loss and in the number of participants achieving a weight loss of $\geq 5 \%{ }^{4}$

Crucial to the present argument regarding semaglutide's place as a weight loss medication, the SUSTAIN 10 trial showed that semaglutide also held superiority over liraglutide, which is currently marketed for weight loss as Saxenda, in overall weight loss, the number of participants achieving weight loss of $\geq 5 \%$, and the number of participants achieving weight loss of $\geq 10 \%$ (table 1). ${ }^{23}$ Of note, subcutaneous semaglutide only requires once-weekly administration, whereas liraglutide is administered once daily. This could provide an option with higher prospects of regimen compliance for patients with obesity. In fact, in most SUSTAIN trials, patients on semaglutide reported better overall treatment satisfaction over comparators. ${ }^{27}$

Along with increased patient satisfaction, the approval of Wegovy as a weight loss medication could result in more avenues to investigate the many underlying mechanisms of weight loss by GLP-1 RAs. Presently, most animal and human studies exploring these mechanisms have been conducted using liraglutide. ${ }^{13}$ With the approval of Wegovy, further studies could be conducted focusing on the mechanisms of GLP-1 RAs in weight loss and studies on combinatorial pharmacotherapeutics in weight loss. Finally, GLP-1 RAs, such as liraglutide and semaglutide, have cardioprotective properties, ${ }^{16}$ which are highly relevant considering that cardiovascular risk factors (such as hypertension) are commonly present in patients with obesity. ${ }^{3}$ GI side effects, including nausea and vomiting, were the most common side effects and led to discontinuation of semaglutide in up to $8 \%$ of the patients (table 2).

\section{SUMMARY OF PIONEER EVIDENCE}

Along with the evidence obtained during the SUSTAIN clinical trial program, a second clinical trial program was run to test the safety and efficacy of oral semaglutide (Rybelsus) as an antidiabetic drug. The PIONEER (Peptide Innovation for Early Diabetes Treatment) clinical trial program focused on obtaining data on glycemic control and weight loss in patients with type 2 diabetes while being treated with oral semaglutide versus comparators. Semaglutide is currently the only oral medication within its drug class, with all other GLP-1 RAs requiring administration via subcutaneous injection. $^{28} 29$ Oral semaglutide is modified by the addition of the absorption enhancer sodium N-(8-[2-hydroxybenzoyl] 
Table 1 Summary of the SUSTAIN results ${ }^{910} 1620-26$

\begin{tabular}{|c|c|c|c|c|}
\hline Trial (n) & Background regiment & Doses and comparators & $\begin{array}{l}\text { Body weight change } \\
\text { from baseline }(\mathrm{kg})\end{array}$ & $\begin{array}{l}\text { Patients with weight } \\
\text { loss of }>5 \%(\%)\end{array}$ \\
\hline \multirow[t]{3}{*}{ SUSTAIN $1(n=388)^{13}$} & \multirow[t]{3}{*}{ Diet and Exercise } & SC semaglutide $0.5 \mathrm{mg}$ & $-3.73^{*}$ & $37^{*}$ \\
\hline & & SC semaglutide $1.0 \mathrm{mg}$ & $-4.53^{*}$ & $45^{*}$ \\
\hline & & Placebo & -0.98 & 7 \\
\hline \multirow[t]{3}{*}{ SUSTAIN $2(n=1231)^{8}$} & \multirow[t]{3}{*}{ Metformin, pioglitazone, rosiglitazone } & SC semaglutide $0.5 \mathrm{mg}$ & $-4.3^{*}$ & $46^{*}$ \\
\hline & & SC semaglutide $1.0 \mathrm{mg}$ & $-6.1^{*}$ & $62^{*}$ \\
\hline & & Sitagliptin $100 \mathrm{mg}$ & -1.9 & 18 \\
\hline \multirow[t]{2}{*}{ SUSTAIN $3(n=809)^{9}$} & \multirow{2}{*}{$\begin{array}{l}\text { Metformin, Sulfonylurea, } \\
\text { Thiazolidinedione }\end{array}$} & SC semaglutide $1.0 \mathrm{mg}$ & $-5.6^{*}$ & $52^{*}$ \\
\hline & & Exenatide ER $2 \mathrm{mg}$ & -1.9 & 17 \\
\hline \multirow[t]{3}{*}{ SUSTAIN $4(n=1089)^{15}$} & \multirow[t]{3}{*}{ Metformin, Sulfonylurea } & SC semaglutide $0.5 \mathrm{mg}$ & $-3.47^{*}$ & $37^{*}$ \\
\hline & & SC semaglutide $1.0 \mathrm{mg}$ & $-5.17^{*}$ & $51^{*}$ \\
\hline & & Insulin glargine & 1.15 & 5 \\
\hline \multirow[t]{3}{*}{ SUSTAIN $5(n=397)^{12}$} & \multirow[t]{3}{*}{ Metformin, Basal insulin } & SC semaglutide $0.5 \mathrm{mg}$ & $-3.7^{*}$ & $42^{*}$ \\
\hline & & SC semaglutide $1.0 \mathrm{mg}$ & $-6.4^{*}$ & $66^{*}$ \\
\hline & & Placebo & -1.4 & 11 \\
\hline \multirow[t]{4}{*}{ SUSTAIN $6(n=3297)^{11}$} & \multirow[t]{4}{*}{$0-2$ antihyperglycemic agents } & SC semaglutide $0.5 \mathrm{mg}$ & $-3.6^{*}$ & NR \\
\hline & & SC semaglutide $1.0 \mathrm{mg}$ & $-4.9^{*}$ & \\
\hline & & Placebo $1.0 \mathrm{mg}$ & -0.7 & \\
\hline & & Placebo $0.5 \mathrm{mg}$ & -0.5 & \\
\hline \multirow[t]{4}{*}{ SUSTAIN $7(n=1201)^{16}$} & \multirow[t]{4}{*}{ Metformin } & SC semaglutide $0.5 \mathrm{mg}$ & $-4.6^{*}$ & $44^{*}$ \\
\hline & & Dulaglutide $0.75 \mathrm{mg}$ & -2.3 & 23 \\
\hline & & $\mathrm{SC}$ semaglutide $1.0 \mathrm{mg}$ & $-2.3^{*}$ & $63^{*}$ \\
\hline & & Dulaglutide $1.5 \mathrm{mg}$ & -1.1 & 30 \\
\hline \multirow[t]{2}{*}{ SUSTAIN $8(\mathrm{n}=788)^{17}$} & \multirow[t]{2}{*}{ Metformin } & SC semaglutide $1.0 \mathrm{mg}$ & $-5.3^{*}$ & 53 \\
\hline & & Canagliflozin $300 \mathrm{mg}$ & -4.2 & 47 \\
\hline \multirow[t]{2}{*}{ SUSTAIN $9(n=302)^{14}$} & \multirow[t]{2}{*}{ Metformin, Sulfonylurea, SGLT-2 inhibitor } & SC semaglutide $1.0 \mathrm{mg}$ & $-4.7^{*}$ & $50^{*}$ \\
\hline & & Placebo & -0.9 & 8 \\
\hline \multirow[t]{2}{*}{ SUSTAIN $10(n=577)^{18}$} & \multirow[t]{2}{*}{ Metformin, SGLT-2 inhibitor } & SC semaglutide $1.0 \mathrm{mg}$ & $-5.8^{*}$ & $56^{*}$ \\
\hline & & Liraglutide $1.2 \mathrm{mg}$ & -1.9 & 18 \\
\hline
\end{tabular}

${ }^{*} \mathrm{P}<0.05$ indicating the the superiority of semaglutide vs the respective comparator.

tAlone or in combination with one another.

ER, extended release; NR, not recorded; SC, subcutaneous; SGLT-2, sodium glucose cotransporter 2; SUSTAIN, Semaglutide Unabated Sustainability in Treatment of Type 2 Diabetes.

amino) caprylate. ${ }^{28} 29$ As an oral medication with low bioavailability, it requires greater doses per tablet; results from the PIONEER clinical trial program indicate that oral semaglutide $7 \mathrm{mg}$ and $14 \mathrm{mg}$ showed more improvement in hemoglobin A1c compared with sitagliptin, but semaglutide $3 \mathrm{mg}$ dose showed no clinically significant benefit. ${ }^{30}$

It was repeatedly demonstrated throughout the PIONEER clinical trials (table 3 ) that the decrease in both HA1c and body weight was significantly greater with oral semaglutide when compared with a placebo. ${ }^{31-35}$ The superiority of oral semaglutide was also observed when compared with other oral antidiabetic medications, such as sitagliptin ${ }^{30} 36$ and empagliflozin. ${ }^{26}$ This superiority was also found in studies comparing oral semaglutide with other agents from the same drug class (dulaglutide ${ }^{37}$ and liraglutide ${ }^{33}{ }^{34}$ ). Similar to the SUSTAIN clinical trials, the PIONEER trials showed improved glycemic control and weight loss with oral semaglutide as an add-on to metformin monotherapy. ${ }^{28} 303233353638$

Oral semaglutide outperformed GLP-1 RA comparatorsdulaglutide and liraglutide-to promote weight loss during the program ${ }^{33-35}$ (table 3). Other successes, such as decreasing waist circumference and BMI of patients, were also observed.
More patients on oral semaglutide achieved a weight loss of at least $5 \%$ and at least $10 \%$ versus those patients being treated with comparators. ${ }^{33}{ }^{36}$ Compared with liraglutide, oral semaglutide showed a greater bodyweight reduction, ${ }^{33}$ which was found later in the program to be dose-dependent, with a $14 \mathrm{mg}$ dose yielding the greatest reductions. ${ }^{34}$ Patients on oral semaglutide have an increased likelihood of achieving weight loss of $5 \%$ or more and $10 \%$ or more when compared with liraglutide (table 3). ${ }^{33}$ The most significant weight loss was established by week $52,{ }^{2836}$ but a significant weight loss was reported as early as 26 weeks. ${ }^{30} 3335$

Regarding safety, oral semaglutide was reported as being non-inferior to treatment with placebo at lowering incidences of major adverse cardiovascular events. ${ }^{28} 38$ Safety of oral semaglutide in patients with moderate renal impairment (glomerular filtration rate (GFR) between 30\% and $59 \%$ ) was also established during PIONEER 5, ${ }^{32}$ with no significant renal changes observed throughout the trial. As in the SUSTAIN trials, oral semaglutide also showed no increased risk of hypoglycemia. ${ }^{30}$ GI adverse effects (table 4), namely mild to moderate nausea and vomiting, were again the most commonly reported. ${ }^{30-35} 37$ Finally, a significant increase in lipase was noted. ${ }^{31}$ 
Table 2 Summary of the SUSTAIN safety outcomes ${ }^{891115-21}$

\begin{tabular}{|c|c|c|c|c|c|c|c|}
\hline Trial (n) & Doses and comparators & $\begin{array}{l}\text { Duration } \\
\text { in weeks }\end{array}$ & AE (\%) & $\begin{array}{l}\text { Discontinuation due } \\
\text { to } \mathrm{AE}(\%)\end{array}$ & $\begin{array}{l}\text { GI AE (\%): severe, } \\
\text { moderate, mild }\end{array}$ & $\begin{array}{l}\text { Discontinuation due } \\
\text { to } \mathrm{GI} A \mathrm{AE}(\%)\end{array}$ & $\begin{array}{l}\text { Severe or } \\
\text { BG-confirmed } \\
\text { hypoglycemia } \\
(\%)\end{array}$ \\
\hline \multirow{3}{*}{$\begin{array}{l}\text { SUSTAIN } 1 \\
(n=388)^{13}\end{array}$} & $\mathrm{SC}$ semaglutide $0.5 \mathrm{mg}$ & \multirow[t]{3}{*}{30} & 64 & 6 & $38:<1,15,30$ & 4 & \multirow[t]{3}{*}{ None } \\
\hline & $\mathrm{SC}$ semaglutide $1.0 \mathrm{mg}$ & & 56 & 5 & $38: 2,13,32$ & 3 & \\
\hline & Placebo & & 53 & 2 & $15: 0,4,12$ & $<1$ & \\
\hline \multirow{3}{*}{$\begin{array}{l}\text { SUSTAIN } 2 \\
(n=1231)^{8}\end{array}$} & SC semaglutide $0.5 \mathrm{mg}$ & \multirow[t]{3}{*}{56} & 75 & 8 & $44: 3,14,37$ & 7 & \multirow[t]{3}{*}{ NR } \\
\hline & $\mathrm{SC}$ semaglutide $1.0 \mathrm{mg}$ & & 71 & 10 & $40: 3,12,35$ & 8 & \\
\hline & Sitagliptin $100 \mathrm{mg}$ & & 72 & 3 & $24: 1,5,20$ & 3 & \\
\hline \multirow{2}{*}{$\begin{array}{l}\text { SUSTAIN } 3 \\
(\mathrm{n}=809)^{9}\end{array}$} & $\mathrm{SC}$ semaglutide $1.0 \mathrm{mg}$ & \multirow[t]{2}{*}{56} & 75 & 9 & NR & NR & \multirow[t]{2}{*}{ two events* } \\
\hline & Exenatide ER $2 \mathrm{mg}$ & & 76 & 7 & & & \\
\hline \multirow{3}{*}{$\begin{array}{l}\text { SUSTAIN } 4 \\
(n=1089)^{15}\end{array}$} & $\mathrm{SC}$ semaglutide $0.5 \mathrm{mg}$ & \multirow[t]{3}{*}{30 weeks } & 70 & 6 & $41: 2,10,36$ & 3 & \multirow[t]{3}{*}{ NR } \\
\hline & $\mathrm{SC}$ semaglutide $1.0 \mathrm{mg}$ & & 73 & 7 & $43: 2,14,38$ & 5 & \\
\hline & Insulin glargine & & 65 & 1 & $15: 1,4,12$ & 0 & \\
\hline \multirow{3}{*}{$\begin{array}{l}\text { SUSTAIN } 5 \\
(n=397)^{12}\end{array}$} & $\mathrm{SC}$ semaglutide $0.5 \mathrm{mg}$ & \multirow[t]{3}{*}{30} & 69 & 5 & \multirow[t]{3}{*}{ NR } & \multirow[t]{3}{*}{ NR } & $8 \%$ \\
\hline & $\mathrm{SC}$ semaglutide $1.0 \mathrm{mg}$ & & 64 & 6 & & & $11 \%$ \\
\hline & Placebo & & 58 & $<1$ & & & $5 \%$ \\
\hline \multirow{4}{*}{$\begin{array}{l}\text { SUSTAIN } 6 \\
(\mathrm{n}=3297)^{11}\end{array}$} & SC semaglutide $0.5 \mathrm{mg}$ & \multirow[t]{4}{*}{104} & 90 & 12 & 51 & \multirow[t]{4}{*}{ NR } & $23 \%$ \\
\hline & SC semaglutide $1.0 \mathrm{mg}$ & & 89 & 15 & 52 & & $22 \%$ \\
\hline & Placebo & & 91 & 6 & 36 & & $22 \%$ \\
\hline & Placebo & & 89 & 8 & 35 & & $21 \%$ \\
\hline \multirow{4}{*}{$\begin{array}{l}\text { SUSTAIN } 7 \\
(\mathrm{n}=1201)^{16}\end{array}$} & $\mathrm{SC}$ semaglutide $0.5 \mathrm{mg}$ & \multirow[t]{4}{*}{40} & 68 & 8 & $43: 3,13,36$ & 5 & $3 \%$ \\
\hline & Dulaglutide $0.75 \mathrm{mg}$ & & 62 & 5 & $33: 1,7,28$ & 2 & $1 \%$ \\
\hline & $\mathrm{SC}$ semaglutide $1.0 \mathrm{mg}$ & & 69 & 10 & $44: 2,16,38$ & 6 & $2 \%$ \\
\hline & Dulaglutide $1.5 \mathrm{mg}$ & & 74 & 7 & $48: 3,13,42$ & 5 & $2 \%$ \\
\hline \multirow{2}{*}{$\begin{array}{l}\text { SUSTAIN } 8 \\
(\mathrm{n}=788)^{17}\end{array}$} & $\mathrm{SC}$ semaglutide $1.0 \mathrm{mg}$ & \multirow[t]{2}{*}{52} & 76 & 10 & 47 & 7 & $2 \%$ \\
\hline & Canagliflozin $300 \mathrm{mg}$ & & 72 & 5 & 28 & 1 & $1 \%$ \\
\hline \multirow{2}{*}{$\begin{array}{l}\text { SUSTAIN } 9 \\
(n=302)^{14}\end{array}$} & $\mathrm{SC}$ semaglutide $1.0 \mathrm{mg}$ & \multirow[t]{2}{*}{30} & 69 & 9 & $37: 1,14,31$ & 7 & $11 \%$ \\
\hline & Placebo & & 60 & 2 & $13: 0,3,11$ & 0 & $2 \%$ \\
\hline \multirow{2}{*}{$\begin{array}{l}\text { SUSTAIN } 10 \\
(\mathrm{n}=577)^{18}\end{array}$} & $\mathrm{SC}$ semaglutide $1.0 \mathrm{mg}$ & \multirow[t]{2}{*}{30} & 71 & 11 & 44 & 7 & \multirow[t]{2}{*}{ NR } \\
\hline & Liraglutide $1.2 \mathrm{mg}$ & & 66 & 7 & 38 & 4 & \\
\hline
\end{tabular}

*The two events of 'Severe or BG-confirmed Hypoglycemia' during SUSTAIN 3 were both in the SC semaglutide $1.0 \mathrm{mg}$ group.

AE, adverse events; BG, blood glucose; ER, extended release; GI, gastrointestinal; NR, not recorded; SC, subcutaneous; SUSTAIN, Semaglutide Unabated Sustainability in Treatment of Type 2 Diabetes.

\section{SUMMARY OF STEP EVIDENCE}

The most recent clinical trial program to investigate the efficacy of subcutaneous semaglutide was the STEP program. The primary goal of the program was to evaluate semaglutide's efficacy as a solely weight loss medication. Participants were recruited based on BMI, excluding those with a diagnosis of type 2 diabetes, ${ }^{11} 13-15$ except STEP 2, which did not exclude patients with type 2 diabetes. ${ }^{12}$ The experimental dose given during the trial was $2.4 \mathrm{mg}$, delivered subcutaneously once a week. The trial program was completed in March 2021 with a total of five trials; however, the results are still pending for STEP 5 (table 5). The results from STEP 1 through 4 showed that semaglutide is superior at weight reduction when compared with placebo. ${ }^{11-14}$ STEP 2 compared 2.4 mg semaglutide with $1.0 \mathrm{mg}$ semaglutide and found $2.4 \mathrm{mg}$ semaglutide to cause more significant weight loss than 1.0 $\mathrm{mg}$ semaglutide. ${ }^{12}$ STEP 4 investigated the discontinuation of semaglutide treatment and found that those who were started on placebo after 20 weeks of treatment with the experimental dose of semaglutide experienced weight gain of around $6 \mathrm{~kg}^{14}$ (table 5). The safety profile of $2.4 \mathrm{mg}$ semaglutide was found to be similar to $1.0 \mathrm{mg}$ subcutaneous semaglutide and oral semaglutide, with mild to moderate GI symptoms being the predominating complaint among participants (table 6). Hypoglycemic events were not frequently reported, an encouraging finding for the administration of semaglutide to patients without type 2 diabetes.

As previously stated, the results from STEP 5 have not been released. A review of these results at a later date would be advisable for future discussions on semaglutide's efficacy. No direct comparison of $2.4 \mathrm{mg}$ semaglutide with other FDA-approved weight loss medications was made during STEP 1 through 5. This would be a fruitful avenue for future research on pharmacotherapeutics for weight reduction.

\section{DEMOGRAPHICS OF SUSTAIN, PIONEER, AND STEP TRIALS}

SUSTAIN 1-10, PIONEER and STEP trials are multicenter, multinational, randomized controlled trials, except PIONEER 9 and 10, conducted in Japan.

Around $60 \%-93 \%$ of the population were white in SUSTAIN 1-10, STEP, and PIONEER 1-8 trials, and about 2\%-10\% were black or Asian. Women accounted 
Table 3 Summary of PIONEER results ${ }^{28} 30-38$

\begin{tabular}{|c|c|c|c|c|}
\hline Trial (n) & Background regiment & Doses and comparators & $\begin{array}{l}\text { Body weight change from } \\
\text { baseline }(\mathrm{kg})\end{array}$ & $\begin{array}{l}\text { Patients with weight } \\
\text { loss of }>5 \%(\%)\end{array}$ \\
\hline \multirow[t]{4}{*}{ PIONEER $1(n=703)^{23}$} & \multirow[t]{4}{*}{ Diet and Exercise } & Oral semaglutide $3 \mathrm{mg}$ & -1.5 & 20 \\
\hline & & Oral semaglutide $7 \mathrm{mg}$ & -2.3 & 27 \\
\hline & & Oral semaglutide $14 \mathrm{mg}$ & $-3.7^{*}$ & $40^{*}$ \\
\hline & & Placebo & -1.4 & 15 \\
\hline \multirow[t]{2}{*}{ PIONEER $2(n=822)^{20}$} & \multirow[t]{2}{*}{ Metformin } & Oral semaglutide $14 \mathrm{mg}$ & -3.8 & 41 \\
\hline & & Empagliflozin $25 \mathrm{mg}$ & -3.7 & 36 \\
\hline \multirow[t]{4}{*}{ PIONEER $3(n=1864)^{22}$} & \multirow[t]{4}{*}{ Metformin, Sulfonylurea } & Oral semaglutide $3 \mathrm{mg}$ & -1.2 & 13 \\
\hline & & Oral semaglutide $7 \mathrm{mg}$ & $-2.2^{*}$ & $19^{*}$ \\
\hline & & Oral semaglutide $14 \mathrm{mg}$ & $-3.1^{*}$ & $30^{*}$ \\
\hline & & Sitagliptin $100 \mathrm{mg}$ & -0.6 & 10 \\
\hline \multirow[t]{3}{*}{ PIONEER $4(n=711)^{25}$} & \multirow[t]{3}{*}{ Metformin, SGLT-2 inhibitor } & Oral semaglutide $14 \mathrm{mg}$ & $-4.4^{*}$ & $44^{*}$ \\
\hline & & Liraglutide $1.8 \mathrm{mg}$ & -3.1 & 28 \\
\hline & & Placebo & -0.5 & 8 \\
\hline \multirow[t]{2}{*}{ PIONEER $5(n=324)^{24}$} & \multirow{2}{*}{$\begin{array}{l}\text { Metformin, Basal insulin, } \\
\text { Sulfonylurea }\end{array}$} & Oral semaglutide $14 \mathrm{mg}$ & $-3.4^{*}$ & $36^{*}$ \\
\hline & & Placebo & -0.9 & 10 \\
\hline \multirow[t]{2}{*}{ PIONEER $6(\mathrm{n}=3183)^{30}$} & \multirow[t]{2}{*}{ No exclusion based on regimen } & Oral semaglutide $14 \mathrm{mg}$ & -4.2 & NR \\
\hline & & Placebo & -0.8 & \\
\hline \multirow[t]{2}{*}{ PIONEER $7(n=504)^{28}$} & \multirow[t]{2}{*}{ 1-2 antihyperglycemic agents } & $\begin{array}{l}\text { Oral semaglutide (flexible dose } 3 \text {, } \\
7, \text { or } 14 \mathrm{mg} \text { ) }\end{array}$ & $-2.6^{*}$ & $27^{*}$ \\
\hline & & Sitagliptin $100 \mathrm{mg}$ & -0.7 & 12 \\
\hline \multirow[t]{4}{*}{ PIONEER $8(n=731)^{27}$} & \multirow{4}{*}{$\begin{array}{l}\text { Metformin, insulin (basal, basal- } \\
\text { bolus, or premixed) }\end{array}$} & Oral semaglutide $3 \mathrm{mg}$ & $-1.4^{*}$ & $13^{*}$ \\
\hline & & Oral semaglutide $7 \mathrm{mg}$ & $-2.4^{*}$ & $31^{*}$ \\
\hline & & Oral semaglutide $14 \mathrm{mg}$ & $-3.7^{*}$ & $39^{*}$ \\
\hline & & Placebo & -0.4 & 3 \\
\hline \multirow[t]{5}{*}{ PIONEER $9(n=243)^{26}$} & \multirow{5}{*}{$\begin{array}{l}\text { Diet and exercise or one } \\
\text { antihyperglycemic agent }\end{array}$} & Oral semaglutide $3 \mathrm{mg}$ & -0.6 & 4 \\
\hline & & Oral semaglutide $7 \mathrm{mg}$ & $-1.1^{*}$ & 10 \\
\hline & & Oral semaglutide $14 \mathrm{mg}$ & $-2.4^{*}$ & $34^{*}$ \\
\hline & & Liraglutide $0.9 \mathrm{mg}$ & 0.0 & 0 \\
\hline & & Placebo & -1.1 & 10 \\
\hline \multirow[t]{4}{*}{ PIONEER $10(n=458)^{29}$} & \multirow[t]{4}{*}{ one antihyperglycemic agent } & Oral semaglutide $3 \mathrm{mg}$ & -0.2 & 5 \\
\hline & & Oral semaglutide $7 \mathrm{mg}$ & $-1.0^{*}$ & $18^{*}$ \\
\hline & & Oral semaglutide $14 \mathrm{mg}$ & $-2.2^{*}$ & $31^{*}$ \\
\hline & & Dulaglutide $0.75 \mathrm{mg}$ & -0.3 & 6 \\
\hline
\end{tabular}

PIONEER (Peptide Innovation for Early Diabetes Treatment) SGLT-2 inhibitor (Sodium Glucose Cotransporter 2 Inhibitors)

${ }^{*} \mathrm{P}<0.05$ indicating the superiority of semaglutide versus the respective comparator.

†Alone or in combination with one another.

$\mathrm{NR}$, not recorded.

for $40 \%-50 \%$ in SUSTAIN $1-10$ and PIONEER $1-8$ trials, 20\%-30\% in PIONEER 9 and 10, but the majority of the study population in the STEP trial were women, accounting for around $50 \%-81 \%$.

In East Asians, beta cell dysfunction is the primary factor causing type 2 diabetes, while insulin resistance and body fat play a minor role compared with Caucasians. Comparison of PIONEERS trials showed that Japanese patients had a more significant reduction in hemoglobin A1c than global trials (PIONEER 1-8). ${ }^{29}$ This finding suggests that diabetes mellitus type 2 in East Asian patients is more likely due to dysfunction of the beta cells than insulin resistance and obesity.

\section{COMPARING AND COMPILING THE EVIDENCE FOR SEMAGLUTIDE}

The superiority of semaglutide in reducing body weight compared with other antidiabetic medications has been relatively well established by the clinical trials discussed above. In the STEP clinical trial, the efficacy of once-weekly semaglutide at a dose of $2.4 \mathrm{mg}$ compared with placebo in the weight reduction of patients with obesity and without diabetes was confirmed. ${ }^{11} 1314$

Here we compared the results reported for semaglutide based on administration modality-oral or subcutaneous injection-based on the PIONEER and SUSTAIN trials' findings (tables 1 and 3). In the PIONEER trial, it was found that a $14 \mathrm{mg}$ dose of oral semaglutide produced a significant weight loss of $2.3 \mathrm{~kg}$, while $0.5 \mathrm{mg}$ and $1.0 \mathrm{mg}$ of subcutaneous semaglutide were associated with $3.73 \mathrm{~kg}$ and $4.53 \mathrm{~kg}$ reduction, respectively. ${ }^{21}{ }^{31}$ Similarly, compared with sitagliptin, both oral semaglutide and subcutaneous semaglutide showed superiority in bodyweight reduction; however, in an indirect comparison of the results (table 1), we found that subcutaneous semaglutide produced even greater bodyweight reduction than oral administration. ${ }^{9} 30$ This trend is observed again when comparing SUSTAIN 8 and 


\begin{tabular}{|c|c|c|c|c|c|c|}
\hline $\begin{array}{l}\text { Trial (number of } \\
\text { participants) }\end{array}$ & Doses and comparators & $\begin{array}{l}\text { Duration in } \\
\text { weeks }\end{array}$ & AE $(\%)$ & $\begin{array}{l}\text { Discontinuation } \\
\text { due to } A E(\%)\end{array}$ & $\begin{array}{l}\text { Discontinuation due } \\
\text { to GI AE (\%) }\end{array}$ & $\begin{array}{l}\text { Severe or BG- } \\
\text { confirmed } \\
\text { hypoglycemia (\%) }\end{array}$ \\
\hline \multirow[b]{4}{*}{ PIONEER $1(\mathrm{n}=703)^{23}$} & Oral semaglutide $3 \mathrm{mg}$ & \multirow[t]{4}{*}{26} & 58 & 2 & 2 & 3 \\
\hline & Oral semaglutide $7 \mathrm{mg}$ & & 53 & 4 & 2 & 1 \\
\hline & Oral semaglutide $14 \mathrm{mg}$ & & 57 & 7 & 5 & 1 \\
\hline & Placebo & & 56 & 2 & 1 & 1 \\
\hline \multirow[b]{2}{*}{ PIONEER $2(\mathrm{n}=822)^{20}$} & Oral semaglutide $14 \mathrm{mg}$ & \multirow[t]{2}{*}{52} & 70 & 11 & 8 & 2 \\
\hline & Empagliflozin $25 \mathrm{mg}$ & & 69 & 4 & 1 & 2 \\
\hline \multirow[b]{4}{*}{ PIONEER $3(n=1864)^{22}$} & Oral semaglutide $3 \mathrm{mg}$ & \multirow[t]{4}{*}{78} & 79 & 6 & 2 & 5 \\
\hline & Oral semaglutide $7 \mathrm{mg}$ & & 78 & 6 & 3 & 5 \\
\hline & Oral semaglutide $14 \mathrm{mg}$ & & 80 & 12 & 7 & 8 \\
\hline & Sitagliptin $100 \mathrm{mg}$ & & 83 & 5 & 3 & 8 \\
\hline \multirow[b]{3}{*}{ PIONEER $4(\mathrm{n}=711)^{25}$} & Oral semaglutide $14 \mathrm{mg}$ & \multirow[t]{3}{*}{52} & 80 & 11 & 8 & 1 \\
\hline & Liraglutide $1.8 \mathrm{mg}$ & & 74 & 9 & 6 & 2 \\
\hline & Placebo & & 67 & 4 & 2 & 2 \\
\hline \multirow[b]{2}{*}{ PIONEER $5(\mathrm{n}=324)^{24}$} & Oral semaglutide $14 \mathrm{mg}$ & \multirow[t]{2}{*}{26} & 74 & 15 & 12 & 6 \\
\hline & Placebo & & 65 & 5 & 2 & 2 \\
\hline \multirow[b]{2}{*}{ PIONEER $6(n=3183)^{30}$} & Oral semaglutide $14 \mathrm{mg}$ & \multirow[t]{2}{*}{ * } & \multirow[t]{2}{*}{ NR } & 12 & 7 & \multirow[t]{2}{*}{ NR } \\
\hline & Placebo & & & 7 & 2 & \\
\hline \multirow[b]{2}{*}{ PIONEER $7(n=504)^{28}$} & $\begin{array}{l}\text { Oral semaglutide (flexible dose } 3 \text {, } \\
7, \text { or } 14 \mathrm{mg} \text { ) }\end{array}$ & \multirow[t]{2}{*}{52} & 78 & 9 & 6 & 6 \\
\hline & Sitagliptin $100 \mathrm{mg}$ & & 69 & 3 & 1 & 6 \\
\hline \multirow[b]{4}{*}{ PIONEER $8(\mathrm{n}=731)^{27}$} & Oral semaglutide $3 \mathrm{mg}$ & \multirow[t]{4}{*}{52} & 74 & 7 & 5 & 28 \\
\hline & Oral semaglutide $7 \mathrm{mg}$ & & 78 & 9 & 7 & 26 \\
\hline & Oral semaglutide $14 \mathrm{mg}$ & & 83 & 13 & 10 & 27 \\
\hline & Placebo & & 76 & 3 & 1 & 29 \\
\hline \multirow{5}{*}{$\begin{array}{l}\text { PIONEER } 9 \\
(n=243)^{26}\end{array}$} & Oral semaglutide $3 \mathrm{mg}$ & \multirow[t]{5}{*}{52} & 76 & 2 & 2 & 0 \\
\hline & Oral semaglutide $7 \mathrm{mg}$ & & 76 & 4 & 2 & 0 \\
\hline & Oral semaglutide $14 \mathrm{mg}$ & & 71 & 0 & 4 & 0 \\
\hline & Liraglutide $0.9 \mathrm{mg}$ & & 67 & 0 & 0 & 4 \\
\hline & Placebo & & 80 & 0 & 0 & 0 \\
\hline \multirow{4}{*}{$\begin{array}{l}\text { PIONEER } 10 \\
(n=458)^{29} \\
(n=458)^{29}\end{array}$} & Oral semaglutide $3 \mathrm{mg}$ & \multirow[t]{4}{*}{52} & 77 & 3 & 31 & 2 \\
\hline & Oral semaglutide $7 \mathrm{mg}$ & & 80 & 6 & 39 & 2 \\
\hline & Oral semaglutide $14 \mathrm{mg}$ & & 85 & 6 & 54 & 3 \\
\hline & Dulaglutide $0.75 \mathrm{mg}$ & & 82 & 3 & 40 & 0 \\
\hline
\end{tabular}

NR means the value was not recorded in the trial results.

*Ongoing study.

AE, adverse events; BG, blood glucose; GI, gastrointestinal; PIONEER, Peptide Innovation for Early Diabetes Treatment.

PIONEER. ${ }^{25} 28$ However, we did not support these comparisons with statistical tests using the original raw data. They, therefore, should not be regarded as definitive conclusions about the superiority of one administration modality of semaglutide in reducing body weight. To our knowledge, no previous studies have compared the differences in the efficacy of oral and subcutaneously injectable semaglutide, which should be addressed in future research. The STEP program did compare $1.0 \mathrm{mg}$ subcutaneous semaglutide and $2.4 \mathrm{mg}$ subcutaneous semaglutide, finding that weight reduction is improved with a dose increase. ${ }^{12}$

Both oral semaglutide and subcutaneous semaglutide showed similar adverse effects (tables 2 and 4), and the most commonly reported were gastrointestinal (primarily mild to moderate nausea and vomiting). Treatment discontinuation due to GI events ranged from $4.9 \%$ to $12 \%$ and from $3 \%$ to $9.4 \%$ during the PIONEER ${ }^{32} 35$ and the SUSTAIN trials, ${ }^{1023}$ respectively. Increased pancreatic lipase was also observed in both clinical trials. ${ }^{12123}$ The incidence of hypoglycemic episodes was low for oral semaglutide ${ }^{30}$ and subcutaneous semaglutide. $^{23} 39$

The only apparent difference between oral semaglutide and subcutaneous semaglutide, besides slight differences in their efficacy in weight reduction, is the route of administration. In a recent study, most patients reported preferring taking oral tablets to subcutaneous injections, supported by the ease of administration and the lack of pain. ${ }^{40}$ From these results, most patients would possibly prefer to take an oral tablet of semaglutide than a once-weekly subcutaneous injection. Currently, the FDA has only given approval to Wegovy, $2.4 \mathrm{mg}$, subcutaneous semaglutide, as an antiobesity medication. ${ }^{7}$ In the future, it may be beneficial to explore oral semaglutide as a weight loss medication, given limited efficacy differences between oral and subcutaneous semaglutide and possible preference toward an oral agent in patient populations. 
Table 5 Summary of STEP results ${ }^{11-15}$

\begin{tabular}{|c|c|c|c|c|}
\hline Trial (n) & Doses and comparators & $\begin{array}{l}\text { Estimated mean weight } \\
\text { change }(\%)\end{array}$ & $\begin{array}{l}\text { Patients with weight loss } \\
\text { of: }>5 \%,>10 \%,>15 \%(\%)\end{array}$ & $\begin{array}{l}\text { Body weight change from } \\
\text { baseline }(\mathrm{kg})\end{array}$ \\
\hline \multirow[t]{2}{*}{ STEP $1(n=1961)^{11}$} & SC semaglutide $2.4 \mathrm{mg}$ & -14.9 & $86.4,69.1,50.5$ & -15.3 \\
\hline & Placebo & -2.4 & $31.5,12.0,4.9$ & -2.6 \\
\hline \multirow[t]{3}{*}{ STEP $2(n=1210)^{12}$} & SC semaglutide $2.4 \mathrm{mg}$ & -9.6 & $68.8,45.6,25.8$ & -9.7 \\
\hline & $\mathrm{SC}$ semaglutide $1.0 \mathrm{mg}$ & -7.0 & 57.1, 28.7, 13.7, & -2.5 \\
\hline & Placebo & -3.4 & $28.5,8.2,3.2$ & -1.3 \\
\hline \multirow[t]{2}{*}{ STEP $3(n=611)^{13}$} & SC semaglutide $2.4 \mathrm{mg}$ & -16 & $86.6,75.3,55.8$ & -16.8 \\
\hline & $\begin{array}{l}\text { Placebo (+intensive behavioral } \\
\text { therapy) }\end{array}$ & -5.7 & $47.6,27,13.2$ & -6.2 \\
\hline \multirow[t]{2}{*}{ STEP $4(n=902)^{14}$} & $\begin{array}{l}\text { SC semaglutide (68 total } \\
\text { weeks) }\end{array}$ & -7.9 & NR & -7.1 \\
\hline & $\begin{array}{l}\text { SC semaglutide ( } 20 \text { weeks), } \\
\text { then placebo ( } 48 \text { weeks) }\end{array}$ & +6.9 & & +6.1 \\
\hline STEP $5(n=304)^{15}$ & $\begin{array}{l}\text { SC semaglutide } 2.4 \mathrm{mg} \\
\text { Placebo }\end{array}$ & Results pending & Results pending & Results pending \\
\hline
\end{tabular}

NR means the value was not recorded in the trial results.

Results for STEP $5^{15}$ are pending at the time of writing this review.

SC, subcutaneous; STEP, Semaglutide Treatment Effect in People with Obesity.

Semaglutide subcutaneous injection is a prefilled pen with only a $4 \mathrm{~mm}, 32$ gauge needle, which makes it patient-friendly and easy to use.

\section{SEMAGLUTIDE VERSUS OTHER WEIGHT LOSS MEDICATIONS}

In the previous sections of this review, we discussed the efficacy of semaglutide in weight loss using the available published data comparing semaglutide with placebo or other antidiabetic medications known to cause weight loss. To adequately address the antiobesity potential of semaglutide, it is essential to consider if at least a $5 \%$ bodyweight reduction is achieved, which is regarded as a clinically significant weight loss parameter. ${ }^{41}$ Therefore, we will compare the results observed in semaglutide compared with other antiobesity medications (without antidiabetic properties), namely phentermine-topiramate, orlistat, and naltrexonebupropion. We will not include lorcaserin (Belviq, Belviq ER), a previously marketed weight loss medication, because the FDA revoked its approval due to the increased risk of malignancies reported in a recent safety clinical trial. ${ }^{6}$

Regarding efficacy data, a meta-analysis of 28 randomized, placebo-controlled clinical trials found that all four antiobesity medications met the FDA weight loss threshold of at least 5\%. Phentermine-topiramate was the most successful medication, followed by liraglutide, as approximately $75 \%$ and $63 \%$ of participants achieved this goal, respectively. ${ }^{42}$ A significant number of participants in each study accomplished a weight loss of at least $10 \%$ compared with a placebo, with phentermine-topiramate and liraglutide showing the best results. ${ }^{42}$

Considering this endpoint, the PIONEER, SUSTAIN, and STEP clinical trials (tables 1 and 3) have shown similar weight loss effects in participants on semaglutide. ${ }^{1120-223138}$ There are no data comparing the impact of semaglutide and phentermine-topiramate on achieving a 5\% weight loss. Comparing the results presented in both trials, we observe that in the primary placebo-controlled trial from the PIONEER program and SUSTAIN 1, around $40 \%$ of participants on semaglutide achieved a weight loss of $5 \%$ or more. ${ }^{21} 31$ Thus, these results could suggest that semaglutide is outperformed by phentermine-topiramate and liraglutide as antiobesity medications. However, the trials testing those two weight loss medications also included other weight loss interventions, such as a hypocaloric diet program $^{43} 44$ or diet and exercise/lifestyle modification

Table 6 Summary of STEP safety outcomes ${ }^{11-15}$

\begin{tabular}{|c|c|c|c|c|c|c|}
\hline Trial (n) & Doses and comparators & $\begin{array}{l}\text { Duration in } \\
\text { weeks }\end{array}$ & $\mathrm{AE}(\%)$ & GI AE (\%) & $\begin{array}{l}\text { Discontinuation } \\
\text { due to GI AE (\%) }\end{array}$ & $\begin{array}{l}\text { Severe or } \\
\text { BG-confirmed } \\
\text { hypoglycemia }\end{array}$ \\
\hline $\begin{array}{l}\text { STEP } 1 \\
(\mathrm{n}=1961)^{11}\end{array}$ & SC semaglutide $2.4 \mathrm{mg}$ Placebo & 68 & 86.786 .4 & 74.247 .9 & 4.50 .8 & 0.60 .8 \\
\hline $\begin{array}{l}\text { STEP } 2 \\
(\mathrm{n}=1210)^{12}\end{array}$ & $\begin{array}{l}\text { SC semaglutide } 2.4 \mathrm{mg} \mathrm{SC} \text { semaglutide } 1.0 \mathrm{mg} \\
\text { Placebo }\end{array}$ & 68 & 97.681 .876 .9 & 63.557 .534 .3 & 4.23 .51 .0 & 5.75 .53 .0 \\
\hline STEP $3(n=611)^{13}$ & $\begin{array}{l}\text { SC semaglutide } 2.4 \mathrm{mg} \text { Placebo (+intensive } \\
\text { behavioral therapy) }\end{array}$ & 68 & 95.896 .1 & 82.863 .2 & 3.40 & 0.50 \\
\hline STEP $4(n=902)^{14}$ & $\begin{array}{l}\text { SC semaglutide ( } 68 \text { total weeks) SC semaglutide } \\
\text { ( } 20 \text { weeks), then Placebo ( } 48 \text { weeks) }\end{array}$ & 68 & 81.375 & 49.126 .1 & 2.42 .2 & 0.61 .1 \\
\hline STEP $5(n=304)^{15}$ & SC semaglutide $2.4 \mathrm{mg}$ Placebo & & Results Pending & Results Pending & Results Pending & Results Pending \\
\hline
\end{tabular}

Results for STEP $5^{15}$ are pending at the time of writing this review.

AE, adverse events; BG, blood glucose; GI, gastrointestinal; SC, subcutaneous; STEP, Semaglutide Treatment Effect in People with Obesity. 
counseling. ${ }^{32} 4546$ The phase III trials of the SUSTAIN and PIONEER programs did not include these additional interventions. The first STEP trial did include lifestyle modifications as part of their experimental requirements and found that $86.5 \%$ of participants on semaglutide achieved a weight loss of at least $5 \% .{ }^{11}$ Therefore, it is reasonable to assume that semaglutide, combined with other interventions, would meet or exceed the accomplishments of liraglutide or even phentermine-topiramate. Studies addressing these comparisons are needed.

Essential differences in safety between these medications should also be considered. As pointed above, semaglutide, like other GLP-1 RAs, is associated with GI adverse effects, namely nausea and vomiting (table 2). More serious adverse effects include pancreatitis and medullary thyroid carcinoma, although thyroid cancers have not been reported in human trials. ${ }^{1}$ Phenterminetopiramate has been associated with severe cardiovascular outcomes, while orlistat intake increased the risk of renal failure and hepatotoxicity. ${ }^{1}$ Semaglutide, on the other hand, lowered death from cardiovascular causes by $26 \%$ when compared with placebo. ${ }^{38}$ Semaglutide does not affect overall renal function and is considered safe for use in patients with moderate renal impairment. ${ }^{32}$ Based on these safety findings, semaglutide could be a better alternative to the antiobesity medications currently on the market. It should also be noted that, as an incretin-based therapy, semaglutide (like other GLP-1 analogs) has a low risk of hypoglycemia.

\section{PRECAUTIONS}

As we described before, the most commonly reported adverse effects of semaglutide during the SUSTAIN clinical trial were GI side effects, including nausea and vomiting. ${ }^{1}$ Patients taking semaglutide experienced these side effects more frequently than comparators (in the SUSTAIN, PIONEER, and STEP trials), but most episodes were transient. ${ }^{4}$ Higher nausea and vomiting rates were experienced at higher doses of semaglutide and lower baseline BMI. ${ }^{4}$ There are also concerns for increased pancreatic lipase and pancreatitis, similar to other GLP-1 RAs. Statistically significant increased lipase was found during both the SUSTAIN and PIONEER trials. ${ }^{20} 31$ One patient in the SUSTAIN 5 trial also developed a metastatic pancreatic carcinoma around 65 days post-treatment. ${ }^{20}$ Treatment with GLP-1 RAs is contraindicated in patients with a history of chronic or idiopathic acute pancreatitis. ${ }^{21}$

A higher incidence of retinopathy complications, including vitreous hemorrhage and blindness, was reported in patients treated with semaglutide compared with placebo. ${ }^{16}$ This was considered to be related to the rapidity and magnitude of glycemic improvement rather than a direct side effect of semaglutide. Gall bladderrelated issues, including cholelithiasis, ranged between $0.2 \%$ and $4.9 \%$, and cardiovascular issues, including tachycardia and arrhythmias, ranged between $1.5 \%$ and 9.8\% in STEP trials 1-4. Other contraindications include a family or personal history of multiple endocrine neoplasia type 2, impaired renal function, or medullary thyroid cancer. These restrictions are based on findings from animal studies. ${ }^{121}$

\section{LIMITATIONS}

This review of literature has few limitations. The three trials from the SUSTAIN clinical trial program-SUSTAIN China multiregional clinical trial, SUSTAIN (Japan), and SUSTAIN (Japan, sitagliptin) - were also excluded due to redundancies in the comparators. ${ }^{17-19}$ This review also includes superficial comparisons of the findings from the SUSTAIN, PIONEER, and STEP trials, as no studies to date have compared the efficacy of subcutaneous and oral semaglutide. These comparisons cannot be considered definitive conclusions due to their indirect nature. A future study comparing these two agents would be a beneficial addition to the literature on the efficacy of semaglutide.

\section{CONCLUSION}

Wegovy (semaglutide) is not only superior at reducing body weight compared with other antidiabetic drugs, but it is also cardioprotective. Another significant benefit of semaglutide is that it can be used for long-term management of weight. With the recent approval of Wegovy, we could expect improvement in patients' weight loss-related outcomes and quality of life.

The high cost of Wegovy can make it unaffordable for many patients unless it is covered by insurance. In addition, this medication is supposed to be used for long-term weight management, which can add high costs to the management of obesity, a growing pandemic. A cost-effectiveness analysis of Wegovy will help clinicians decide if it should be preferred compared with other weight loss drugs. However, a long list of insurance and prescription benefit plans can make it impossible to determine if the medication is covered, which can be another barrier.

Twitter Gurdeep Singh @SinghHotmail

Acknowledgements We thank Our Lady of Lourdes Memorial Hospital and Lake Erie College of Osteopathic Medicine for their support.

Contributors All authors have substantially participated in the preparation and agree to be accountable for all aspects of the work related to the manuscript. GS, MK, and MB-E contributed to the conception, literature search, writing, editing, revision, and final approval of the manuscript.

Funding The authors have not declared a specific grant for this research from any funding agency in the public, commercial or not-for-profit sectors.

Competing interests None declared.

Patient consent for publication Not required.

Provenance and peer review Not commissioned; externally peer reviewed.

Open access This is an open access article distributed in accordance with the Creative Commons Attribution Non Commercial (CC BY-NC 4.0) license, which permits others to distribute, remix, adapt, build upon this work noncommercially, and license their derivative works on different terms, provided the original work is properly cited, an indication of whether changes were made, and the use is non-commercial. See: http://creativecommons.org/ licenses/by-nc/4.0/.

\section{ORCID iD}

Gurdeep Singh http://orcid.org/0000-0001-6044-7419

\section{REFERENCES}

1 Isaacs D, Prasad-Reddy L, Srivastava SB. Role of glucagon-like peptide 1 receptor agonists in management of obesity. AM J Health-Syst Pharm 2016;73:1493-507.

2 World Health Organization. Obesity and overweight, 2020. Available: https:// www.who.int/news-room/fact-sheets/detail/obesity-and-overweight

3 Ladenheim E. Liraglutide and obesity: a review of the data so far. Drug Des Devel Ther 2015;9:1867-75. 
4 Ahrén B, Atkin SL, Charpentier G, et al. Semaglutide induces weight loss in subjects with type 2 diabetes regardless of baseline BMI or gastrointestinal adverse events in the SUSTAIN 1 to 5 trials. Diabetes Obes Metab 2018;20:2210-9.

5 Matheus ASdeM, Tannus LRM, Cobas RA, et al. Impact of diabetes on cardiovascular disease: an update. Int J Hypertens 2013;2013:653789-15.

6 U. S. Food and Drug Administration. FDA requests the withdrawal of weightloss drug Belviq, Belviq XR (Lorcaserin) from the market: potential risk of cancer outweighs the benefits. Washington, DC Center for Drug Evaluation and Research; 2020

7 U. S. Food and Drug Administration. FDA approves new drug treatment for chronic weight management, first since 2014. Washington, DC Center for Drug Evaluation and Research; 2021.

8 Blundell J, Finlayson G, Axelsen M, et al. Effects of once-weekly semaglutide on appetite, energy intake, control of eating, food preference and body weight in subjects with obesity. Diabetes Obes Metab 2017;19:1242-51.

9 Ahrén B, Masmiquel L, Kumar H, et al. Efficacy and safety of once-weekly semaglutide versus once-daily sitagliptin as an add-on to metformin thiazolidinediones, or both, in patients with type 2 diabetes (SUSTAIN 2): a 56-week, double-blind, phase 3a randomised trial. Lancet Diabetes Endocrinol 2017;5:341-54.

10 Ahmann AJ, Capehorn M, Charpentier G, et al. Efficacy and safety of onceweekly semaglutide versus exenatide ER in subjects with type 2 diabetes (SUSTAIN 3): a 56-week, open-label, randomized clinical trial. Diabetes Care 2018;41:258-66.

11 Wilding JPH, Batterham RL, Calanna S, et al. Once-weekly semaglutide in adults with overweight or obesity. N Engl J Med 2021;384:989-1002.

12 Davies M, Færch L, Jeppesen OK. Semaglutide 2.4 mg once a week in adults with overweight or obesity, and type 2 diabetes (STEP 2): a randomised, double-blind, double-dummy, placebo-controlled, phase 3 trial. Lancet Diabetes Endocrinol 2021;397:971-84.

13 Wadden TA, Bailey TS, Billings LK, et al. Effect of subcutaneous semaglutide vs placebo as an adjunct to intensive behavioral therapy on body weight in adults with overweight or obesity: The STEP 3 randomized clinical trial. JAMA 2021;325:1403-13.

14 Rubino D, Abrahamsson N, Davies M, et al. Effect of continued weekly subcutaneous semaglutide vs placebo on weight loss maintenance in adults with overweight or obesity: The STEP 4 randomized clinical trial. JAMA 2021;325:1414-25

15 Kushner RF, Calanna S, Davies M, et al. Semaglutide $2.4 \mathrm{mg}$ for the treatment of obesity: key elements of the STEP trials 1 to 5. Obesity 2020;28:1050-61.

16 Marso SP, Bain SC, Consoli A, et al. Semaglutide and cardiovascular outcomes in patients with type 2 diabetes. N Engl J Med 2016;375:1834-44.

17 Liong J, Dong X, Freddy E. Efficacy and safety of once-weekly semaglutide vs. once-daily sitagliptin as add-on to metformin in subjects with type 2 diabetes (SUSTAIN China MRCT): a 30-week double-blind, phase 3a, randomised trial. Diabetes 2020;69(supplement 1.

18 Yutaka S, Yasuo T, Takeshi O, et al. Safety and efficacy of semaglutide onceweekly vs sitagliptin once-daily, both as monotherapy in Japanese people with type 2 diabetes. Diabetes Obes Metab 2018;20:378-88.

19 Kohei K, Yuichira Y, Jirotaka W, et al. Safety and efficacy of once-weekly semaglutide vs additional oral antidiabetic drugs in Japanese people with inadequately controlled type 2 diabetes: a randomized trial. Diabetes Obes Metab 2018:20:1202-12.

20 Rodbard HW, Lingvay I, Reed J, et al. Semaglutide added to basal insulin in type 2 diabetes (SUSTAIN 5): a randomized, controlled trial. J Clin Endocrinol Metab 2018;103:2291-301

21 Sorli C, Harashima S-ichi, Tsoukas GM, et al. Efficacy and safety of once-weekly semaglutide monotherapy versus placebo in patients with type 2 diabetes (SUSTAIN 1): a double-blind, randomised, placebo-controlled, parallelgroup, multinational, multicentre phase 3a trial. Lancet Diabetes Endocrinol 2017;5:251-60.

22 Zinman B, Bhosekar V, Busch R, et al. Semaglutide once weekly as add-on to SGLT-2 inhibitor therapy in type 2 diabetes (SUSTAIN 9): a randomised placebo-controlled trial. The Lancet Diabetes Endocrinol 2019;7:356-67.

23 Aroda VR, Bain SC, Cariou B, et al. Efficacy and safety of once-weekly semaglutide versus once-daily insulin glargine as add-on to metformin (with or without sulfonylureas) in insulin-naive patients with type 2 diabetes (SUSTAIN 4): a randomised, open-label, parallel-group, multicentre, multinational, phase 3a trial. Lancet Diabetes Endocrinol 2017;5:355-66.

24 Pratley RE, Aroda VR, Lingvay I, et al. Semaglutide versus dulaglutide onceweekly in patients with type 2 diabetes (SUSTAIN 7): a randomised, open-label, phase 3b trial. Lancet Diabetes Endocrinol 2018:6:275-86.

25 Lingvay I, Catarig A-M, Frias JP, et al. Efficacy and safety of once-weekly semaglutide versus daily canagliflozin as add-on to metformin in patients with type 2 diabetes (SUSTAIN 8): a double-blind, phase 3b, randomised controlled trial. Lancet Diabetes Endocrinol 2019;7:834-44.

26 Capehorn MS, Catarig A-M, Furberg JK, et al. Efficacy and safety of onceweekly semaglutide $1.0 \mathrm{mg}$ vs once-daily liraglutide $1.2 \mathrm{mg}$ as add-on to 1-3 oral antidiabetic drugs in subjects with type 2 diabetes (SUSTAIN 10). Diabetes Metab 2020;46:100-9

27 Jendle J, Birkenfeld AL, Polonsky WH, et al. Improved treatment satisfaction in patients with type 2 diabetes treated with once-weekly semaglutide in the SUSTAIN trials. Diabetes Obes Metab 2019:21:2315-26.

28 Rodbard HW, Rosenstock J, Canani LH, et al. Oral semaglutide versus empagliflozin in patients with type 2 diabetes uncontrolled on metformin: the PIONEER 2 trial. Diabetes Care 2019:42:2272-81.

29 Thethi TK, Pratley R, Efficacy MJJ. Safety and cardiovascular outcomes of once-daily oral semaglutide in patients with type 2 diabetes: the PIONEER programme. Diabetes Obes Metab2020:1-15.

30 Rosenstock J, Allison D, Birkenfeld AL, et al. Effect of additional oral semaglutide vs sitagliptin on glycated hemoglobin in adults with type 2 diabetes uncontrolled with metformin alone or with sulfonylurea. JAMA 2019;321:1466-80

31 Aroda VR, Rosenstock J, Terauchi Y, et al. PIONEER 1: randomized clinical trial of the efficacy and safety of oral semaglutide monotherapy in comparison with placebo in patients with type 2 diabetes. Diabetes Care 2019;42:1724-32.

32 Mosenzon O, Blicher TM, Rosenlund S, et al. Efficacy and safety of ora semaglutide in patients with type 2 diabetes and moderate renal impairment (PIONEER 5): a placebo-controlled, randomised, phase 3A trial. Lancet Diabetes Endocrinol 2019;7:515-27

33 Pratley R, Amod A, Hoff ST, et al. Oral semaglutide versus subcutaneous liraglutide and placebo in type 2 diabetes (PIONEER 4): a randomised, doubleblind, phase 3A trial. Lancet 2019:394:39-50.

34 Yamada Y, Katagiri H, Hamamoto Y, et al. Dose-response, efficacy, and safety of oral semaglutide monotherapy in Japanese patients with type 2 diabetes (PIONEER 9): a 52-week, phase 2/3a, randomised, controlled trial. Lancet Diabetes \& Endocrinology 2020:8:377-91.

35 Zinman B, Aroda VR, Buse JB, et al. Efficacy, safety, and tolerability of oral semaglutide versus placebo added to insulin with or without metformin in patients with type 2 diabetes: the PIONEER 8 trial. Diabetes Care 2019:42:2262-71

36 Pieber TR, Bode B, Mertens A, et al. Efficacy and safety of oral semaglutide with flexible dose adjustment versus sitagliptin in type 2 diabetes (PIONEER 7): a multicentre, open-label, randomised, phase 3A trial. Lancet Diabetes Endocrinol 2019;7:528-39.

37 Yabe D, Nakamura J, Kaneto $\mathrm{H}$, et al. Safety and efficacy of oral semaglutide versus dulaglutide in Japanese patients with type 2 diabetes (PIONEER 10): an open-label, randomised, active-controlled, phase 3A trial. Lancet Diabetes Endocrinol 2020:8:392-406.

38 Husain M, Birkenfeld AL, Donsmark M, et al. Oral semaglutide and cardiovascular outcomes in patients with type 2 diabetes. N Engl J Med 2019;381:841-51.

39 DeVries JH, Desouza C, Bellary S, et al. Achieving glycaemic control without weight gain, hypoglycaemia, or gastrointestinal adverse events in type 2 diabetes in the SUSTAIN clinical trial programme. Diabetes Obes Metab 2018;20:2426-34

40 Quante M, Thate-Waschke I, Schofer M. What are the reasons for patient preference? A comparison between oral and subcutaneous administration. Z Orthop Unfall 2012;150:397-403.

41 Center for Drug Evaluation and Research. Guidance for industry developing products for weight management, 2007. Available: http://www.fda.gov/ucm/ groups/fdagov-public/@fdagov-drugs-gen/documents/document/ucm071612. pdf [Accessed 22 July 2020].

42 Khera R, Murad MH, Chandar AK. Association of pharmacological treatments for obesity with weight loss and adverse events: a systematic review and metaanalysis. JAMA 2016:315:2424-34.

43 Apovian CM, Aronne L, Rubino D, et al. A randomized, phase 3 trial of naltrexone SR/bupropion SR on weight and obesity-related risk factors (CORII). Obesity 2013;21:935-43.

44 Finer N, James WPT, Kopelman PG, et al. One-year treatment of obesity: a randomized, double-blind, placebo-controlled, multicentre study of orlistat, a gastrointestinal lipase inhibitor. Int J Obes 2000;24:306-13.

45 Allison DB, Gadde KM, Garvey WT, et al. Controlled-release phentermine/ topiramate in severely obese adults: a randomized controlled trial (EQUIP). Obesity 2012;20:330-42

46 Fidler MC, Sanchez M, Raether B, et al. A one-year randomized trial of lorcaserin for weight loss in obese and overweight adults: the BLOSSOM trial. $J$ Clin Endocrinol Metab 2011:96:3067-77. 